\title{
LARVAS DE SIDERONE MARTHESIA NEMESIS (ILLIGER) (LEPIDOPTERA, NYMPHALIDAE, CHARAXINAE) EM CERRADO DE BRASÍLIA, DISTRITO FEDERAL, BRASIL
}

\author{
H.C. Morais ${ }^{1}$ \\ I.R. Diniz ${ }^{2}$ \\ J.R. Silva ${ }^{2}$
}

\begin{abstract}
CATERPILLARS OF SidERONE MARTHESIA NEMESIS (ILliger) (LEPIDOPTERA, Nymphalidae, Charaxinae) in Cerrado near Brasilia, Distrito Federal, Brazil. Caterpillars of Siderone marthesia nemesis (Illiger, 1802) (Nymphalidae, Charaxinae) have been found on Casearia sylvestris Sw. (Flacourtiaceae), during the second half of the rainy season in an area of cerrado near Brasília, Distrito Federal, Brazil. They occurred at very low density and are cryptic at all instars. The pupal stage does not occur on the host plant. Average duration of the pupa under laboratory conditions is 14.4 days.

KEY WORDS. Charaxinae, Siderone, hostplant, crypsis
\end{abstract}

Siderone marthesia (Cramer, [1777]) (Nymphalidae, Charaxinae) ocorre do Brasil até o México, pertence ao grupo de borboletas cujos adultos se alimentam de frutos fermentados e as plantas hospedeiras utilizadas pelo gênero são da família Flacourtiaceae (DEVRIES 1987).

Siderone marthesia nemesis (Illiger, 1802) ocorre no Brasil central e os adultos são mais freqüentes no cerrado, mas ocorrem também em trilhas na mata de galeria e em área de sucessão (PINHEIRO \& ORTIZ 1992).

Larvas de Siderone marthesia nemesis foram encontradas em uma área de cerrado sensu stricto da Fazenda Água Limpa (Universidade de Brasília) utilizando Casearia sylvestris $\mathrm{Sw}$. (Flacourtiaceae) como planta hospedeira (DINIZ \& MORAIS 1995). C. sylvestris é um arbusto que raramente ultrapassa um metro de altura e é comum nesta área.

As larvas são solitárias e estão presentes em $C$. sylvestris na segunda metade da estação de chuvas. As larvas foram encontradas em março/92, fevereiro e março de 1993 e 1994 e em março/95.

Em 1994 foram examinados 538 indivíduos de Casearia sylvestris à procura de larvas de Siderone (Tab. I). A freqüência de larvas em C. sylvestris é muito baixa e estas ocorrem em folhas maduras mesmo quando a planta hospedeira apresenta folhas novas. Das 20 larvas coletadas em todo esse período apenas uma estava em folha nova.

1) Departamento de Ecologia, Universidade de Brasília. 70910-900 Brasília, Distrito Federal, Brasil.

2) Departamento de Zoologia, Universidade de Brasília. 70910-900 Brasília, Distrito Federal, Brasil. 
Tabela I. Freqüência de ocorrência de larvas de Siderone marthesia nemesis em Casearia sylvestris num cerrado de Brasília, com informações sobre o ínstar e a posição das larvas nas folhas. Nervura = ponta da nervura central; limbo = superficie superior da folha; entre parênteses $=$ número de larvas.

\begin{tabular}{|c|c|c|c|c|c|c|}
\hline \multirow{2}{*}{ Data } & \multicolumn{2}{|c|}{ Número de plantas } & \multirow{2}{*}{$\begin{array}{c}\text { Porcentagem } \\
\text { plantas com } \\
\text { larvas }\end{array}$} & \multicolumn{3}{|c|}{ Instar e posição das larvas nas folhas } \\
\hline & Examinadas & Com larvas & & Instares iniciais & Instares finais & Último instar \\
\hline $10-11-94$ & 52 & 0 & & & & \\
\hline $23-11-94$ & 171 & 2 & 1,2 & & Nervura (1) & Limbo (1) \\
\hline $02-111-94$ & 90 & 1 & 1,1 & & & Nervura (1) \\
\hline $25-111-94$ & 85 & 2 & 2,4 & & Nervura (1) & Limbo (1) \\
\hline $29-111-94$ & 140 & 6 & 4,3 & Nervura (2) & Nervura (3) & Limbo (1) \\
\hline Total & 538 & 11 & 2,1 & & & \\
\hline
\end{tabular}

As larvas dos primeiros ínstares se alimentam do ápice das folhas mantendo intacta a nervura central à qual são acrescentadas fezes e fragmentos de folha presos com fios de seda (Fig. 1a). Este local é denominado de substrato de fixação ou poleiro (FurTADo 1984; CASAgRANDE \& MielKe 1985). As larvas permanecem nesta ponta de nervura tanto no período de alimentação como no de repouso (Tab. I). Nesta posição elas estão bem camufladas e são difíceis de serem percebidas. As larvas dos últimos ínstares também permanecem na nervura, principalmente quando em repouso, mas são mais facilmente percebidas (Fig. 1b).

As larvas de último ínstar (Fig. lc) permanecem, tanto para alimentação quanto para repouso, sobre o limbo foliar. Quando em repouso são muito semelhantes a uma folha seca enrolada. H.C. Morais coletou a primeira larva de Siderone ao tentar remover uma "folha seca" que estava sobre C. sylvestris. As larvas maduras de $S$. marthesia, na Costa Rica, têm uma mancha branca conspícua no mesotorax (DEVRIES 1987) o que não ocorre nas larvas de S. marthesia nemesis (Fig. 1c).

Em laboratório o tempo médio de pupa foi de 14,4 dias $(s=2,07 ; n=5$ ) (Fig. 1d). Durante todo o período de coleta não foi encontrada nenhuma pupa nas plantas hospedeiras. Uma pupa de Siderone foi encontrada em Qualea parviflora Mart. (Vochysiaceae) em uma área de cerrado do Jardim Botânico de Brasília (C.M. Dias, comunicação pessoal). Eunica bechina (Hewitson), outro ninfalídeo encontrado em cerrados de São Paulo, empupa em arbustos próximos à planta hospedeira (OLIVEIRA \& FREITAS 1991) o que pode ocorrer também com Siderone.

Uma das larvas coletadas no campo e criada em laboratório estava parasitada por um Chalcidoidea (Hymenoptera) ainda não identificado, e o parasitóide emergiu na fase de pupa. Na Costa Rica as larvas de S. marthesia também são atacadas por um calcidoideo (DEVRIES 1987).

A baixa freqüência de plantas com larvas de Lepidoptera parece ser uma característica do cerrado (ANDRADE et al. 1995; PRICE et al. 1995) bem como a baixa freqüência de larvas de uma mesma espécie de Lepidoptera. Siderone marthesia nemesis mantem esse padrão com apenas 11 larvas encontradas em 538 plantas examinadas. A captura de adultos com iscas também não revelou grande abundância desta espécie (PINHEIRO \& ORTIZ 1992). 

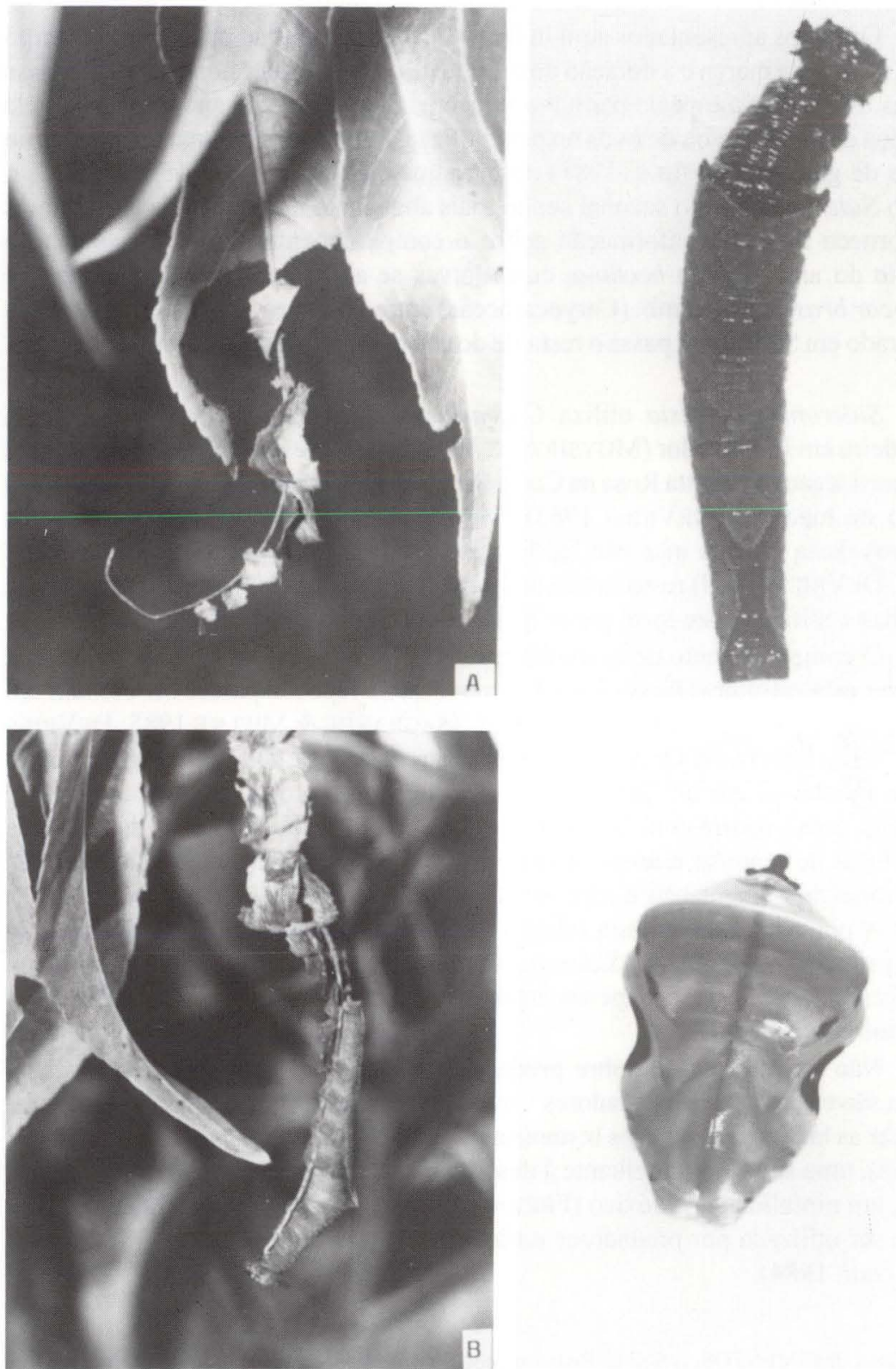

Fig. 1. Siderone marthesia nemesis (Nymphalidae, Charaxinae) em Casearia sylvestris (Flacourtiaceae), em um cerrado da Fazenda Água Limpa (Brasília, Distrito Federal). (a) Folha comida mostrando a nervura central intacta e o acúmulo de fragmentos de folha; (b) larva de instares finais em posição de repouso na nervura central de uma folha; (c) larva de último instar; (d) pupa. 
Os dados apresentados aqui indicam que as larvas estão presentes no campo entre fevereiro e março e a duração do estágio de pupa em laboratório não ultrapassa 20 dias. Assim, se a espécie permanece na área durante todo o ano isso se dá pela presença de adultos e/ou de ovos ou pela utilização de um habitat mais úmido como a mata de galeria. DEVRIES (1987) observa que em floresta seca da Costa Rica o gênero Siderone é muito sazonal sendo mais abundante na estação de chuvas mas não fornece nenhuma informação sobre o comportamento destas borboletas no restante do ano. Eunica bechina, cujas larvas se alimentam de folhas novas de Caryocar brasiliense Camb. (Caryocaraceae) entre setembro e janeiro em uma área de cerrado em São Paulo, passa o restante do ano como adulto (FREITAS \& OLIVEIRA 1992).

Siderone marthesia utiliza Casearia sylvestris e C. nitida como planta hospedeira em El Salvador (MUYSHONDT 1976), e C. sylvestris e Zuelania quidonia (Flacourtiaceae) em Santa Rosa na Costa Rica, alimentando-se tanto de folhas novas quanto de maduras (DEVRIES 1985). MUYSHONDT (1976) descreve os estágios imaturos desta espécie mas não faz nenhum comentário sobre a camuflagem das larvas. DEVRIES (1987) ressalta que as larvas de Siderone acumulam fezes na ponta de folhas e utilizam este local para repouso quando não estão se alimentando.

O comportamento de acumular fezes na forma de uma estrutura organizada e utilizar esta estrutura (frass chains) como local de repouso parece ser comum em Charaxinae (Freitas \& Oliveira 1992; CASAgrande \& Mielke 1985; DeVries 1985, 1987). FREITAS \& OLIVEIRA (1992) sugerem que este comportamento permite à larva manter-se em um local com pouco acesso de predadores, especialmente formigas, como ocorre com larvas de Heliconiini que utilizam pontas de gavinhas como local de repouso e áreas recortadas e semi-isoladas do limbo foliar (ilhas) como local de alimentação e repouso (BENSON et al. 1976; BENTLEY \& BENSON 1988). A ponta seca da nervura foliar e o acúmulo de fezes torna o local utilizado pelos primeiros ínstares de Siderone semelhante aos citados acima e com um patrulhamento de formigas apenas esporádico, especialmente em uma planta sem nectários extra florais.

Não há informação sobre predação em larvas de Siderone mas elas sem dúvida são crípticas para predadores visualmente orientados. Um coletor aprende a procurar as larvas de primeiros ínstares a partir do tipo de dano encontrado na folha (Fig. 1a), uma situação semelhante à descrita para Asterocampa idyja argus (Bates, 1864), um ninfalídeo do México (FRIEDLANDER 1985). Esta característica também parece ser utilizada por predadores para localizar lagartas (HEINRICH 1979, 1993; REAL et al. 1984).

AGRADECIMENTOS. A C.E.G. Pinheiro pela identificação do lepidóptero, S. Scherrer por informações sobre as larvas em 1995, I. Andrade e V.O. Becker por informações bibliográficas, F.G. Menezes pelas fotografias e a dois revisores anônimos pelos comentários enriquecedores no primeiro manuscrito. J.R. Silva recebeu bolsa do CNPq (Proc. $\mathrm{n}^{\circ}$ 501025/92-8). 


\section{REFERÊNCIAS BIBLIOGRÁFICAS}

ANDRADE, I.; I.R. DiNIZ \& H.C. MORAIS 1995. A lagarta de Cerconota achatina (Oecophoridae, Stenomatinae): biologia e ocorrência em plantas hospedeiras do gênero Byrsonima (Malpighiaceae). Revta bras. Zool. 12 (4): 735-741.

BENSON, W.W.; K.S. Brown \& L.E. GILBERT. 1976. Coevolution of plants and herbivores: passion flower butterflies. Evolution 29: 659-680.

BENTLEY, B.L. \& W.W. BENSON. 1988. The influence of ant foraging patterns on the behavior of herbivores, p.297-306. In: J.C. TRAGER (Ed.). Advances in myrmecology. New York, E.J. Brill, 601p.

Casagrande, M.M. \& O.H.H. MielKe. 1985. Estágios imaturos de Agrias claudina claudianus Staudinger (Lepidoptera, Nymphalidae, Charaxinae). Revta bras. Ent. 29: 139-142.

DEVRIES, P.J. 1985. Hostplant records and natural history notes on Costa Rican butterflies (Papilionidae, Pieridae \& Nymphalidae). J. Res. Lepid. 24 (4): 290-333.

1987. The butterflies of Costa Rica and their natural history Papilionidae, Pieridae, Nymphalidae. Princeton, Princeton University Press, 327p.

DINIZ, I.R. \& H.C. MORAIS. 1995. Larvas de Lepidoptera e suas plantas hospedeiras em um cerrado de Brasília, DF, Brasil. Revta bras. Ent. 39 (4): 755-770.

FreitAS, A.V.L. \& P.S. OliveIRA. 1992. Biology and behavior of the neotropical butterfly Eunica bechina (Nymphalidae) with special reference to larval defense against ant predation. J. Res. Lepid. 31 (1-2): 1-11.

FRIEDLANDER, T.P. 1985. The biology and morphology of the immature stages of Asterocampa idyja argus (Bates) (Lepidoptera: Nymphalidae). J. Res. Lepid. 24 (3): 209-225.

FURTADO, E. 1984. Contribuição ao conhecimento dos Lepidoptera brasileiros. - I. Biologia de Agrias amydon ferdinandi Fruhstorfer (Nymphalidae, Charaxinea). Revta bras. Ent. 28 (3): 289-294.

HEINRICH, G.H. 1979. Foraging strategies of caterpillars: leaf damage and possible predator avoidance strategies. Oecologia 42: 325-337.

1993. How avian predators constrain caterpillar foraging, p.224-247. In: N.E. STAMP \& T.M. CASEY (Eds). Caterpillars - ecological and evolutionary constraints on foraging. New York, Chapman \& Hall, 587p.

MUYSHONDT, A. 1976. Notes on the life cycle and natural history of butterflies of El Salvador. VII. Archaeoprepona antimache gulina, Siderone marthesia, Zaretis callidryas and Consul electra (Nymphalidae). J. Lepid. Soc. 30 (3): 159-168.

OliveirA, P.S. \& A.V.L. FreitAs. 1991. Hostplant record for Eunica bechina magnipunctata (Nymphalidae) and observation on oviposition sites and immature biology. J. Res. Lepid. 30 (1-2): 140-141.

PINHEIRO, C.E.G \& J.V.C. ORTIZ. 1992. Communities of fruit-feeding butterflies along a vegetation gradient in central Brazil. J. Biogeogr. 19: 505-511. 
PRICE, P.W.; I.R. DINIZ; H.C. MoraIS \& E.S.A. MARQUES. 1995. The abundance of insect herbivore species in the tropics: the high local richness of rare species. Biotropica 27 (4): 468-478.

Real, R.G.; R. IANazZi; A.C. Kamil \& B. HeInRICH. 1984. Discrimination and generalization of leaf damage by blue jays (Cyanocitta cristata). Anim. Learn. Behav. 12: 202-208.

Recebido em 05.XI.1995; aceito em 30.VIII.1996. 